\title{
The effects of payout and probability magnitude on the Allais paradox
}

\author{
BETHANY J. WEBER \\ Rutgers University, New Brunswick, New Jersey
}

\begin{abstract}
The Allais paradox decision bias was first offered as a challenge to the expected utility theory over 60 years ago. Although the Allais paradox is a standard challenge for normative theories of risky choice, its causes are not well understood. The present experiment uses two manipulations of the Allais paradox to investigate the commonly proposed probability-weighting explanation of the paradox. Reducing the magnitude of the outcomes did not affect the size of the Allais paradox, contradicting previous literature and supporting the probability weighting hypothesis. Reducing the probability of the nonzero outcomes to eliminate certainty reduced, but did not eliminate, the Allais paradox, a result inconsistent with probability weighting and other theories of the Allais paradox. The results suggest that the certainty effect alone cannot explain the Allais paradox.
\end{abstract}

The goal of most decisions is to find the option that leads to the best possible outcome, but the outcomes of decisions are often not known in advance. One proposed axiom of rational choice under conditions of uncertainty is the independence axiom, which states that any outcome common to two sets of options should not influence the choice between the two options (Savage, 1954).

Although highly intuitive, the independence axiom is the subject of a famous challenge proposed by Allais in 1953. When presented with the pairs of gambles shown in Table 1, decision makers tend to choose the safe common consequence (CC)-high gamble and the risky CC-low gamble: They prefer a $100 \%$ chance of $\$ 1$ million to the 3 -outcome risky gamble, but prefer the $10 \%$ chance of $\$ 5$ million to the $11 \%$ chance of $\$ 1$ million (Allais, 1953).

To see why these preferences violate the independence axiom, suppose these gambles are to be resolved by drawing a ball from an urn with 100 balls numbered 1 to 100 , as shown in Table 2. When making a choice between the first pair of gambles, the only question should be your preferences when drawing Balls 1-11. Because winning $\$ 1$ million on a draw of Ball 12-100 is common to both options - a common consequence - how you feel about it is irrelevant to the decision. Moreover, changing the common consequence from $\$ 1$ million to $\$ 0$ (as in the second pair of gambles) should not change the decision, since the outcome of drawing Balls 1-11 has not changed (Savage, 1954).

This violation of the independence axiom is commonly known as the Allais paradox, a robust and widely demonstrated phenomenon (e.g., Birnbaum, 2004; Camerer, 1989; Conlisk, 1989; Kahneman \& Tversky, 1979; Slovic \& Tversky, 1974; Wu \& Gonzalez, 1996).

\section{Explanations of the Allais Paradox}

The most commonly proposed explanations for the Allais paradox involve probability-weighting functions. Examples of theories which explain the Allais paradox (and many other decision biases) via the shape of a probability-weighting function include prospect theory (PT) (Kahneman \& Tversky, 1979); cumulative prospect theory (Tversky \& Kahneman, 1992); and rank-dependent expected utility (Quiggin, 1982).

Under PT, a widely used probability-weighting theory, the value of a gamble with probability $p$ of winning outcome $X$ is $\pi(p) U(X)$. Here $\pi$ is a probability-weighting function that overweights small values of $p$ and underweights large values of $p$, and $\mathrm{U}$ is a utility function assigning a utility to the monetary outcome of the gamble. Because $\pi(0)=0$ and $\pi(1)=1$, the fact that the $\pi$ overweights small probabilities and underweights large ones means that the $\pi$ function changes very rapidly (and is possibly discontinuous) near 0 and 1 .

According to PT, the Allais paradox occurs because of the steepness of the $\pi$ function near the endpoints. When decision makers look at the risky option in the CC-high gamble pair, they overweight the $1 \%$ chance of winning $\$ 0$, treating it as if it were much more likely than it actually is. This makes the risky option seem unacceptably high, compared with the certain win of \$1 million in the safe CC-high option. However, in the CC-low gamble pair, both the $90 \%$ probability of $\$ 0$ in the risky option and the $89 \%$ probability in the safe option fall on a comparatively flat region of the $\pi$ function. The $1 \%$ difference in the probability of losing seems much smaller than it did in the CC-high condition and receives less weight, leading the decision maker to choose the gamble with the highest

B. J.Weber, weber@biac.duke.edu 
Table 1

Examples of the Allais Paradox (Word Problem Format)

\begin{tabular}{|c|c|c|c|}
\hline \multicolumn{4}{|c|}{ Choice one (CC-high) } \\
\hline Risky & $10 \%-\$ 5$ million & $89 \%-\$ 1$ million & $1 \%-\$ 0$ \\
\hline Safe & \multicolumn{3}{|c|}{$100 \%-\$ 1$ million } \\
\hline \multicolumn{4}{|c|}{ Choice two (CC-low) } \\
\hline Risky & $10 \%-\$ 5$ million & $90 \%-\$ 0$ & \\
\hline Safe & $11 \%-\$ 1$ million & $89 \%-\$ 0$ & \\
\hline
\end{tabular}

Note-CC-high, common consequence high; CC-low, common consequence low.

Table 2

Examples of the Allais Paradox (Matrix Format)

\begin{tabular}{|c|c|c|c|}
\hline & Balls 1-10 & Ball 11 & Balls $12-100$ \\
\hline \multicolumn{4}{|c|}{ Choice one (CC-high) } \\
\hline Risky & $\$ 5$ million & $\$ 0$ & $\$ 1$ million \\
\hline Safe & $\$ 1$ million & $\$ 1$ million & $\$ 1$ million \\
\hline \multicolumn{4}{|c|}{ Choice two (CC-low) } \\
\hline Risky & $\$ 5$ million & $\$ 0$ & $\$ 0$ \\
\hline Safe & $\$ 1$ million & $\$ 1$ million & $\$ 0$ \\
\hline
\end{tabular}

Note-CC-high, common consequence high; CC-low, common consequence low.

expected value. A typical PT $\pi$ function and its role in the Allais paradox is illustrated in Figure 1.

\section{Small Outcomes and the Allais Paradox}

In the present experiment, I examined two possible challenges to the probability-weighting explanation of the Allais paradox. The first challenge involves the size of the monetary outcomes used in the paradox. The hypothetical outcomes used to demonstrate the Allais paradox are usually on the order of thousands or even millions of dollars, much larger sums than an undergraduate generally deals with. Attempts to demonstrate the Allais paradox for outcomes similar to what most people deal with on a dayto-day basis have had mixed results. Some studies have demonstrated the Allais paradox using outcomes of less than a few hundred dollars (e.g., Birnbaum, 2004, 2007), although others have found the Allais paradox is greatly reduced or eliminated for small outcomes (e.g., Camerer, 1989; Chew \& Waller, 1986; Fan, 2002).

All of the probability-weighting theories mentioned above incorporate one and only one $\pi$ function; that is, under these theories the weights received by each probability are constant across all sizes of payout. If the Allais paradox is driven by the shape of the $\pi$ function, changes to the size of the outcomes should not produce changes to the paradox, as long as the relationships between the outcome magnitudes remain the same. Thus, past findings that the Allais paradox is reduced or eliminated for small outcomes are inconsistent with the probability-weighting explanation proposed by PT.

However, it is possible that past failures to find the Allais paradox for small outcomes may indicate only that the paradox is harder to detect for small outcomes, not that it does not occur. Decision makers are generally more riskseeking for gambles with small outcomes than they are for gambles with large outcomes (e.g., Green, Myerson, \& Ostaszewski, 1999; Rachlin, Brown, \& Cross, 2000; Weber \& Chapman, 2005b). This increase in risk-seeking may mean that the Allais paradox is more difficult to detect for small outcomes than for large ones.

The possible decreased ability to detect the Allais paradox for small outcomes is due to techniques generally used to study the paradox. All studies that have examined the Allais paradox for small outcomes in the past have done so by asking the subjects to simply choose between pairs of gambles; subjects demonstrated the paradox by choosing the safe CC-high gamble and the risky CC-low gamble. However, the important feature of the Allais paradox is not the pattern of choices themselves but the change in risk attitudes that the choices represent; that is, increased risk-seeking after the common consequence is removed. Two single-choice pairs can only detect the Allais paradox if the gambles used are chosen so that the change in risk preference actually produces a preference reversal between the two gambles. A decision maker who is sufficiently risk-seeking may choose the risky payout in both gambles, even if he is actually more risk-seeking for the CC-low gambles than he is for the CC-high gambles (and thus still experiencing the Allais paradox). A subject risk-seeking enough to choose the risky CC-high gamble cannot show the Allais paradox, using a single-choice technique, even if the underlying change in risk preference is present. There is no third "extra-risky" option in the CC-low gamble that could be chosen to show this increase in risk-seeking; there is only the standard risky CC-low gamble, which makes it appear that no change in risk-seeking has occurred.

This problem can be expected to occur more often for small outcomes than for large ones: Because subjects tend to be more risk-seeking for small outcomes, they are more likely to choose the risky CC-high gamble when outcomes are small. Thus the Allais paradox may only appear to be eliminated for small outcomes when, actually, subjects simply cannot demonstrate it for small outcomes using the choice technique. Supporting this hypothesis, multiple studies on the Allais paradox and small outcomes reported a greater percentage of subjects choosing the risky CChigh gamble for small outcomes than for large ones, although none report whether the difference is significant

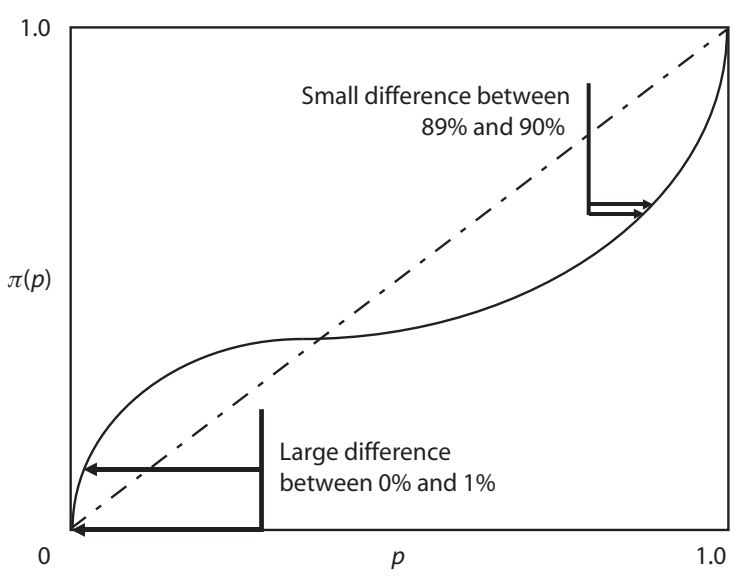

Figure 1. The $\pi$ function and the Allais paradox. 
(Birnbaum, 2007; Camerer, 1989; Chew \& Waller, 1986; Fan, 2002).

If the Allais paradox is really eliminated for small outcomes - that is, removing a common consequence does not increase risk-seeking for small outcomes-this is strong evidence against the traditional probabilityweighting explanation of the paradox. However, if failures to find the Allais paradox for small outcomes are due to an inability to detect the paradox rather than to an actual elimination of the paradox, these results do not contradict the $\pi$ function explanation of the paradox. One way to address this issue is to abandon choice in favor of a more sensitive technique. One commonly used alternative to choice is matching, in which subjects adjust one of the outcomes in a pair of gambles until they are indifferent between the two gambles. Although there is a large body of evidence to suggest that decision makers do not always display consistent preferences when comparing choice responses to matching responses (e.g., Lichtenstein \& Slovic, 1971; Slovic \& Lichtenstein, 1983; Tversky, Slovic, \& Kahneman, 1990), this issue does not arise when matching responses are compared with other matching responses, and several decision biases (including the Allais paradox) have been successfully demonstrated this way (e.g., Weber \& Chapman, 2005a, 2005b). Thus, the more sensitive matching technique can be used to detect whether the Allais paradox is reduced or eliminated for small outcomes.

\section{Probability and the Allais Paradox}

The probability-weighting explanation of the Allais paradox depends on the steepness of the $\pi$ function near the endpoints: the large difference between a $0 \%$ and $1 \%$ chance of an undesirable outcome and, equivalently, the large difference between $99 \%$ chance and a $100 \%$ chance of a desirable outcome.

An obvious question is: What happens to the Allais paradox when probabilities are so altered that they no longer include the endpoints of the $\pi$ function? If the Allais paradox is indeed the result of a special attractiveness of certainty (a certainty effect), reducing the probability of all the outcomes so that none of the gambles include certainty should eliminate the paradox.

Previous literature suggests that the Allais paradox can be eliminated by reducing the probability of the nonzero outcomes to eliminate certainty (Camerer, 1992; Conlisk, 1989; Harless, 1992; Sopher \& Gigliotti, 1993). However, all of these studies altered the probability of the outcomes in a rather unusual manner: They added and subtracted percentage points from the various probabilities. This alteration makes sense in the context in which the experiments were originally performed, to test hypotheses related to the Marschak-Machina probability triangle (Machina, 1982; Marschak, 1950), a discussion of which is beyond the scope of this paper. However, taken out of that theoretical framework, such a manipulation is an odd one; although it eliminates the large change from $0 \%$ to $1 \%$, it does not really accomplish the goal of moving the probabilities away from the endpoints of the $\pi$ function.
Although the function is steepest between $0 \%$ and $1 \%$ and between $99 \%$ and $100 \%$, it is still thought to be quite steep within a few percentage points of the endpoints (Kahneman \& Tversky, 1979); nudging the gambles by a percentage point or two does not remove the outcomes from this region. In fact, such a nudge increases the number of outcomes with very small probabilities by causing previously impossible outcomes to become merely improbable ones. Moreover, it alters one important aspect of the basic structure of the Allais paradox: In the classic Allais paradox, the entire common consequence is shifted from the middle outcome to the lowest outcome. In a nudged gamble, only part of the common consequence of the gamble is shifted to the lowest outcome. It is not clear what effect, if any, this change in the problem structure has on the Allais paradox.

Another way to reduce the probability of the Allais paradox outcomes is simply to divide the probability of all the nonzero outcomes by a common ratio. This approach preserves the structure of the Allais paradox, since the entire common consequence is shifted to the lowest outcome. It also avoids outcomes with very small or very large probabilities, but rather moves all outcomes into the middle of the $\pi$ function. To my knowledge, this approach to reducing the probability of the Allais paradox has not been previously attempted in the literature. A pilot study (Weber, Marks, \& Chapman, 2003) suggested that decreasing the nonzero outcomes of a set of Allais paradox gambles by $50 \%$ reduced, but did not eliminate, the Allais paradox. If this result is replicable, it poses significant problems for the probability-weighting explanation of the Allais paradox, which predicts that the Allais paradox should not occur under such circumstances.

The purpose of the present experiment was twofold. The first purpose was to test the probability-weighting explanation of the Allais paradox by examining the effects on the paradox of reducing the probability of all nonzero outcomes. If the Allais paradox persisted when all the probabilities involved fell in the flat, middle region of the $\pi$ function, it would be evidence that probability-weighting theories might not be the correct explanation for the Allais paradox. The second purpose was to determine whether the Allais paradox could be found for small outcomes using a matching technique. If the Allais paradox was not found for small outcomes, even when the more sensitive matching technique was used, this would also be evidence against the probabilityweighting explanation.

The structure of the present experiment is somewhat unusual. Two experiments were conducted on separate groups of subjects, differing only in that the second experiment used greater reductions in payout and probability than did the first. Although these two waves of testing were conducted on different groups of subjects, because both waves involved extremely similar tasks and are directed to answer the same basic questions, I am discussing them together: First, I discuss the results of the payout manipulations for both waves, followed by the results of the probability manipulations for both waves. 


\section{METHOD}

\section{Subjects}

As part of a class requirement for an introductory psychology class, 406 Rutgers University undergraduates participated, 203 in Wave 1 and 203 in Wave 2. No subject participated in both waves of the experiment.

\section{Design}

Each subject saw both levels of the Allais paradox; that is, both gamble pairs with a common consequence representing a monetary gain (CC-high gambles) and those with a common consequence of $\$ 0$ (CC-low gambles). The primary method of preference elicitation was choice titration, a form of matching in which an indifference point was obtained for each subject via a series of choices. To facilitate comparison with other experiments in the literature, I also included 8 single-choice problems in each of the waves. Both choice titration and single-choice problems are described in more detail below.

\section{Preference Elicitation}

Choice titration. In the present experiment, subjects matched the two gambles in each pair (CC-high or CC-low) by adjusting the highest of the three outcomes of the risky gamble. With this technique, any significant difference between the subject's indifference point for the CC-high gambles and his indifference point for the CC-low gambles indicates the Allais paradox. Subjects' indifference points were elicited using a computerized choice-titration procedure, in which the subject made a repeated series of choices between the risky and safe gambles of an Allais paradox pair. For the initial choice in the titration procedure, the highest outcome of the risky gamble was set to the midpoint of a predefined interval that established the minimum and maximum possible values for the indifference point. In subsequent choices, the highest outcome was adjusted toward the subject's indifference point, in response to the subject's previous choices. These choices followed a bisection algorithm, in which each choice eliminated half the possible values for the highest outcome. The subject was presented with the midpoint of a series of successively smaller intervals, until the value for the highest outcome that made the subject indifferent between the safe and risky gambles was obtained to the desired degree of accuracy. This algorithm has been used to elicit matching responses in a number of prior experiments (e.g., Chapman \& Weber, 2006; Du, Green, \& Myerson, 2002; Myerson, Green, Hanson, Holt, \& Estle, 2003; Weber \& Chapman, 2005a).

As mentioned above, use of the bisection algorithm requires an initial maximum and minimum value to be set. I used $\$ 25,000$ ( $\$ 25$ in the $1 / 1,000$ condition, $\$ 5$ in the $1 / 5,000$ condition) as the initial minimum, because if the highest outcome were $\$ 25,000$, the risky gamble would be strictly dominated by the safe one. The larger the initial interval, the more choices are required to obtain the same degree of accuracy. Although it is desirable to have a large enough interval to encompass all possible indifference points, too large an interval will make the experiments excessively long and increase risk of noise due to subject fatigue. I chose a value I believed to be higher than most indifference points as the high end of the starting interval: $\$ 100,000$ for the large-outcome gambles, $\$ 100$ for the $1 / 1,000$ condition gambles, and $\$ 20$ for the $1 / 5,000$ condition gambles. Any indifference points higher than the maximum starting value must be grouped together. The bisection process continued for 5 choices, after which the midpoint of the remaining interval was considered the indifference point of the subject. The error around this indifference point was $3.125 \%$ of the size of the initial interval.

Check choices. To eliminate incorrect indifference points caused by subject inattention or mistakes, the subject was presented with two check choices after the indifference point was obtained. These questions used values slightly above and below the indifference point and were not distinguished from the other questions in the series. If the subject's response to these questions was not consistent with the calculated indifference point, a message box was displayed informing the subject of an inconsistent response, and the sequence of choices was started over. (The message box was included because, in earlier experiments using this technique without a message box, subjects wondered why the questions were being repeated.) Each question was repeated until the subject produced a consistent series of responses, or until the question had been presented three times. Questions for which subjects failed to give a consistent series of responses after three repetitions were not used in the analysis.

Single choice. Single-choice questions were presented to the subject following the choice-titration questions. These questions were similar to the single-choice questions used in previous literature demonstrating the Allais paradox: They consisted of a single choice for each pair (the CC-high pair and the CC-low pair), with a value for the highest outcome determined by the mean of the indifference points obtained for the CC-high pair and the CC-low pair in the earlier choice-titration questions. This is the value for the highest outcome that maximizes the likelihood of showing the Allais paradox using single choice. If the subject showed the Allais paradox in the choice-titration questions, the mean of the CC-high and CC-low indifference points would be lower than the CC-high indifference point (resulting in the subject choosing the safe CC-high gamble) and higher than the CC-low indifference point (resulting in the subject choosing the risky CC-low gamble). The single-choice questions were presented in a visually identical manner to the choice-titration questions, and nothing was done to enable the subjects to distinguish between the choice-titration and single-choice questions.

\section{Experimental Manipulations}

Outcome magnitude. The outcome-magnitude manipulation included two levels of outcome magnitude: a large outcome and a small outcome. In the large-outcome gambles, the lowest outcome was $\$ 0$, the middle outcome was $\$ 25,000$, and the highest outcome's value varied according to the choice-titration procedure described above. In the first wave of the experiment, the small-outcome gamble outcomes were equal to those for the large-outcome gambles divided by 1,000 (the 1/1,000 condition); in the second, they were equal to those for the large-outcome gambles divided by 5,000 (the $1 / 5,000$ condition). Thus, the outcome-magnitude manipulation was conducted in a 2 (common consequence present or absent) $\times 2$ (outcome magnitude) within-subjects design. The gambles used in the present experiment are shown in Table 3.

Subjects also saw six pairs of single-choice problems. For the large-outcome single-choice pairs, the highest outcome was equal to the average of the indifference points found for the CC-high and CClow large-outcome choice titration problems. The small-outcome single-choice pairs were obtained in the same manner, using the small-outcome indifference points. A second pair of small-outcome single-choice gambles, obtained by dividing the outcomes of the large-outcome single-choice gamble by either 1,000 (Wave 1) or 5,000 (Wave 2) was included for greater similarity to previous literature, in which small-outcome choices were generally obtained by dividing the large-outcome payouts by a common amount.

Probability magnitude. For both the choice-titration problems and the single-choice problems, subjects saw two levels of probability magnitude: large probability and small probability. To produce the small-probability gambles (both choice titration and single choice) the probability associated with each nonzero payout in the large probability gambles was divided by a common value. In Wave 1 , the probabilities were divided by 2 to produce the small probability condition (the $50 \%$ condition); in Wave 2 they were divided by 10 (the $10 \%$ condition). Thus, the probability manipulation was conducted in a 2 (common consequence present or absent) $\times 2$ (probability magnitude) within-subjects design (see Table 3 ). The probabilitymagnitude manipulation also included two pairs of single choices, a large-probability choice and a small-probability choice. These were obtained from the mean of the indifference points obtained during the corresponding choice-titration pairs in the manner described for the outcome-magnitude manipulation above. 
Table 3

The Gambles Used in the Present Experiment

\begin{tabular}{|c|c|c|c|}
\hline Payout/Probability & $\begin{array}{c}\text { Common } \\
\text { Consequence }\end{array}$ & Risky Option & Safe Option \\
\hline \multirow[t]{2}{*}{ Large, Both Waves } & High & $\begin{array}{l}10 \% \text { chance of } \$ \\
89 \% \text { chance of } \$ 25,000 \\
1 \% \text { chance of } \$ 0\end{array}$ & $100 \%$ chance of $\$ 25,000$ \\
\hline & Low & $\begin{array}{l}10 \% \text { chance of } \$ \\
90 \% \text { chance of } \$ 0\end{array}$ & $\begin{array}{l}11 \% \text { chance of } \$ 25,000 \\
89 \% \text { chance of } \$ 0\end{array}$ \\
\hline \multirow[t]{2}{*}{ Small, Wave $1(1 / 1,000)$} & High & $\begin{array}{l}10 \% \text { chance of } \$ \\
89 \% \text { chance of } \$ 25 \\
1 \% \text { chance of } \$ 0\end{array}$ & $100 \%$ chance of $\$ 25$ \\
\hline & Low & $\begin{array}{l}10 \% \text { chance of } \$ \\
90 \% \text { chance of } \$ 0\end{array}$ & $\begin{array}{l}11 \% \text { chance of } \$ 25 \\
89 \% \text { chance of } \$ 0\end{array}$ \\
\hline \multirow[t]{2}{*}{ Small, Wave $2(1 / 5,000)$} & High & $\begin{array}{l}10 \% \text { chance of } \$ \\
89 \% \text { chance of } \$ 5 \\
1 \% \text { chance of } \$ 0\end{array}$ & $100 \%$ chance of $\$ 5$ \\
\hline & Low & $\begin{array}{l}10 \% \text { chance of } \$ \\
90 \% \text { chance of } \$ 0\end{array}$ & $\begin{array}{l}11 \% \text { chance of } \$ 5 \\
89 \% \text { chance of } \$ 0\end{array}$ \\
\hline \multirow[t]{2}{*}{ Small, Wave $1(1 / 2)$} & High & $\begin{array}{l}5 \% \text { chance of } \$ \\
44.5 \% \text { chance of } \$ 25,000 \\
50.5 \% \text { chance of } \$ 0\end{array}$ & $\begin{array}{l}50 \% \text { chance of } \$ 25,000 \\
50 \% \text { chance of } \$ 0\end{array}$ \\
\hline & Low & $\begin{array}{l}5 \% \text { chance of } \$ \\
95 \% \text { chance of } \$ 0\end{array}$ & $\begin{array}{l}5.5 \% \text { chance of } \$ 25,000 \\
94.5 \% \text { chance of } \$ 0\end{array}$ \\
\hline \multirow[t]{2}{*}{ Small, Wave $2(1 / 10)$} & High & $\begin{array}{l}1 \% \text { chance of } \$ \\
8.9 \% \text { chance of } \$ 25,000 \\
90.1 \% \text { chance of } \$ 0\end{array}$ & $\begin{array}{l}10 \% \text { chance of } \$ 25,000 \\
90 \% \text { chance of } \$ 0\end{array}$ \\
\hline & Low & $\begin{array}{l}1 \% \text { chance of } \$ \\
99 \% \text { chance of } \$ 0\end{array}$ & $\begin{array}{l}1.1 \% \text { chance of } \$ 25,000 \\
98.9 \% \text { chance of } \$ 0\end{array}$ \\
\hline
\end{tabular}

All told, each wave of the experiment had a total of six choicetitration problems: two high-probability small-outcome gambles (used in the outcome-magnitude analysis only); two low-probability large-outcome gambles (used in the probability magnitude analysis only); and two high-probability large-outcome gambles (used in both analyses). There were also eight single-choice questions: two each of two types of high-probability small-outcome pairs (outcome magnitude analysis); two low-probability large-outcome pairs (probability magnitude analysis); and two high-probability largeoutcome pairs (both analyses).

\section{Materials}

Questions were presented to the subjects on their own computers via the World Wide Web. Subjects read through a series of instruction pages before starting the experiment. The choices were presented one at a time in a separate window. The instructions were available to the subjects at all times during the experiment. The order of the gambles was determined randomly with the restriction that, because the single choice gambles were generated using data from the choice titration gambles, they had to appear after the choice titration gambles. Only 4 of 203 subjects took in excess of $15 \mathrm{~min}$ to complete the experiment in Wave 1 (mean, with these 4 subjects excluded $=6.37 \mathrm{~min}$ ) and no subjects took over $15 \mathrm{~min}$ in Wave 2 (mean $=6.25 \mathrm{~min}$ ). An analysis with the 4 outlier subjects excluded did not differ from the full analysis and is not reported.

\section{RESULTS}

Because of the similarity between the two waves of the experiment, the results are discussed together and are organized according to the two main questions the experiment addressed. First, I will discuss the results of the payout manipulations across both waves of testing, for both choice titration and single choice. This is followed by presentation and discussion of the results of the probability manipulations.

\section{Distributions}

The distribution of the indifference points was not normal for either wave of the experiment. Overall, the distributions were bimodal, with a large peak at the floor of the distribution and a small peak at the ceiling. The percentage and number of responses at floor and ceiling are shown in Table 4.

A response at floor indicates strong risk-seeking behavior, a common pattern: In many instances, subjects seemed not to care about the $1 \%$ difference between the chance of winning the risky gamble and that of winning the safe gamble, provided the risky gamble had a higher payout. A response at ceiling indicates strong risk aversion; that is, the subject prefers the safe gamble, no matter what. This response pattern was most common for the CChigh gambles. (The number of responses at floor was also smallest for these gambles.) The fact that this very strong

Table 4

Payout Manipulation: Percentage of Responses at Floor/Ceiling

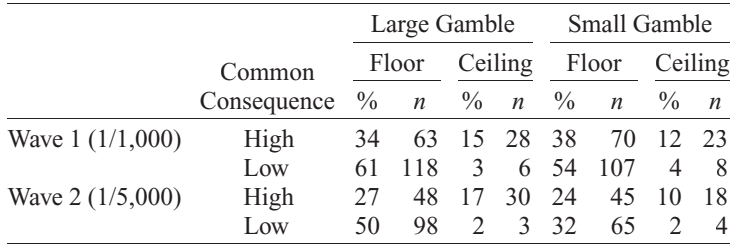


preference for the safe gamble was most often expressed when the safe gamble was certain is consistent with a probability-weighting explanation of the Allais paradox, in which certainty is overweighted.

I reanalyzed the indifference point data after eliminating all responses at either floor or ceiling. These results did not differ from the results of the analyses using the full data set, except where noted and are not reported.

Because the indifference point distributions were not normal, I also ran a nonparametric Friedman test on the size of the Allais paradox (the difference between the CC-high and CC-low indifference points) and Wilcoxon/ Mann-Whitney tests on the indifference points. These results did not differ from the parametric tests except where noted and are not reported.

To obtain values for the percentage of explainable variance accounted for by each factor, I also fitted multilevel mixed models to the data. Again, these results did not differ from those of the repeated-subjects ANOVA, except where noted and are not reported.

\section{Outcome Magnitude}

Choice titration. The mean indifference points for the gambles used in the payout magnitude manipulation are shown in Table 5. The "normalized large gamble" values shown in Table 5 are the mean indifference points for the large gambles divided by either 1,000 (Wave 1) or 5,000 (Wave 2). These values are presented for easier comparison between the large-outcome gamble and small-outcome gamble indifference points.

In each wave of the experiment, 203 subjects provided four indifference points, resulting in 812 data points. In the first wave of the experiment, 50 data points $(6 \%)$ were missing due to failed check questions. The missing values came from 33 subjects. In the second wave of the experiment, 58 data points ( $7 \%$ ) were missing due to failed check questions. The missing check questions came from a total of 40 subjects. The missing observations from both waves were simply excluded from the analyses. As there were no check questions for the single choices, it was impossible for single-choice responses to produce missing values.

A larger indifference point for the highest outcome indicated that the subject demanded larger possible winnings to compensate for the $1 \%$ chance of winning nothing in the risky gamble. Thus, higher indifference points indicate greater preference for the safe gamble and higher levels of risk aversion. If the indifference points are higher for the CC-high gambles than for the CC-low ones, this indicates a shift in risk preference between the two gambles - that is, the Allais paradox.

As shown in Table 5, the indifference points are higher for the CC-high gambles than for the CC-low gambles, for both the large and small gambles. A 2 (common consequence present or absent) $\times 2$ (outcome magnitude) ANOVA found that the main effect of common consequence was significant $[F(1,192)=86.27, p<.0001$, Wave $1 ; F(1,193)=141.02, p<.0001$, Wave 2], indicating that the Allais paradox was significant. However, the main effect of outcome magnitude was not significant $[F(1,196)=1.07, p=.30$, Wave $1 ; F(1,194)=0.03, p=$ .87 , Wave 2]. The interaction between outcome magnitude and common consequence was also not significant $[F(1,169)=0.66, p=.42$, Wave $1 ; F(1,162)=3.14, p=$ .07 , Wave 2]. ${ }^{1}$ Fitting a multilevel model indicated that in Wave $1,18.8 \%$ of the explainable within-subjects variance was explained by the effects of common consequence, and $0.01 \%$ by the effects of payout magnitude. For Wave 2, $26.4 \%$ of the explainable within-subjects variance was explained by the effects of common consequence, whereas the model including only payout magnitude was worse than a model with no predictors. ${ }^{2}$

The lack of a main effect of outcome magnitude indicates that subjects were not more risk-seeking for smalloutcome gambles than they were for large-outcome ones. The lack of an interaction indicates that the Allais paradox was not significantly smaller for small-outcome gambles than it was for large-outcome ones, though both the nonparametric test and the multilevel model found a small effect of payout magnitude on the size of the Allais paradox for the 1/5,000 gambles.

Single choice. The percentage of subjects preferring the risky option for the single choices is shown in Table 6. In both waves, subjects showed the Allais paradox: The main effect of common consequence was significant $\left[\chi^{2}(1, N=203)=46.50, p<.0001\right.$, Wave $1 ; \chi^{2}(1, N=$ $203)=47.62, p<.0001$, Wave 2$]$. The interaction be-

Table 5

Payout Manipulation: Mean (With Standard Deviation) and Median (Med) Indifference Values for Highest Outcome, Before (Top) and After (Bottom) Responses at Floor and Ceiling Were Removed

\begin{tabular}{|c|c|c|c|c|c|c|c|c|c|c|}
\hline & \multirow{3}{*}{$\begin{array}{l}\text { Common } \\
\text { Consequence }\end{array}$} & \multicolumn{9}{|c|}{ Gamble } \\
\hline & & \multicolumn{3}{|c|}{ Large Outcome } & \multicolumn{3}{|c|}{ Normalized Large } & \multicolumn{3}{|c|}{ Small Outcome } \\
\hline & & $M$ & $S D$ & Med & $M$ & $S D$ & Med & $M$ & $S D$ & Med \\
\hline \multirow[t]{2}{*}{ Wave $1(1 / 1,000)$} & High & 51,558 & 29,988 & 30,859 & 51.56 & 29.99 & 30.86 & 48.36 & 28.60 & 30.86 \\
\hline & Low & 34,081 & 17,985 & 26,172 & 34.08 & 17.99 & 26.17 & 33.69 & 16.12 & 26.17 \\
\hline \multirow[t]{3}{*}{ Wave $2(1 / 5,000)$} & High & 57,826 & 30,249 & 43,750 & 11.57 & 6.05 & 8.75 & 10.91 & 5.52 & 8.98 \\
\hline & Low & 34,554 & 16,934 & 27,344 & 6.91 & 3.39 & 5.47 & 7.46 & 3.33 & 6.17 \\
\hline & & \multicolumn{9}{|c|}{ No Floor/Ceiling } \\
\hline \multirow[t]{2}{*}{ Wave $1(1 / 1,000)$} & High & 54,524 & 25,661 & 44,921 & 54.52 & 25.66 & 44.92 & 52.58 & 24.82 & 40.23 \\
\hline & Low & 41,753 & 19,287 & 30,859 & 41.75 & 19.29 & 30.86 & 37.15 & 12.42 & 30.86 \\
\hline \multirow[t]{2}{*}{ Wave $2(1 / 5,000)$} & High & 60,778 & 25,024 & 56,640 & 12.16 & 5.00 & 11.33 & 11.70 & 4.69 & 11.80 \\
\hline & Low & 41,172 & 18,588 & 30,859 & 8.23 & 3.71 & 6.17 & 8.20 & 3.04 & 7.11 \\
\hline
\end{tabular}


Table 6

Payout Manipulation: Percentage of Subjects Choosing the Risky Gamble

\begin{tabular}{|c|c|c|c|c|c|c|c|}
\hline & \multirow{3}{*}{$\begin{array}{c}\text { Common } \\
\text { Consequence }\end{array}$} & \multicolumn{6}{|c|}{ Gamble } \\
\hline & & \multicolumn{2}{|c|}{ Large } & \multicolumn{2}{|c|}{$\begin{array}{c}\text { Small } \\
\text { Derived }\end{array}$} & \multicolumn{2}{|c|}{$\begin{array}{c}\text { Small } \\
\text { Matched }\end{array}$} \\
\hline & & $\%$ & $n$ & $\%$ & $n$ & $\%$ & $n$ \\
\hline \multirow[t]{2}{*}{ Wave $1(1 / 1,000)$} & High & 62 & 126 & 55 & 112 & 53 & 108 \\
\hline & Low & 87 & 177 & 75 & 153 & 77 & 156 \\
\hline \multirow[t]{2}{*}{ Wave $2(1 / 5,000)$} & High & 57 & 115 & 54 & 109 & 58 & 117 \\
\hline & Low & 84 & 171 & 72 & 147 & 74 & 150 \\
\hline
\end{tabular}

tween common consequence and outcome size was significant $\left[\chi^{2}(1, N=203)=4.58, p=.03\right.$, Wave $1 ; \chi^{2}(1$, $N=203)=8.16, p=.004$, Wave 2], meaning the Allais paradox was smaller for the small-outcome gambles than for the large-outcome gambles. However, the Allais paradox was still significant for the small-outcome gambles $\left[\chi^{2}(1, N=203)=38.96, p<.0001\right.$, Wave $1 ; \chi^{2}(1, N=$ $203)=28.14, p<.0001$, Wave 2].

Outcome magnitude summary. The purpose of the outcome-magnitude manipulations in the present experiment was to see if the Allais paradox would occur for small outcomes when a technique more sensitive than the standard single choice technique was used. These results suggest that the Allais paradox does indeed occur for small outcomes. Responses generated by the choice-titration technique not only showed a significant Allais paradox for small outcomes, but an Allais paradox that was not significantly smaller for small outcomes than it was for large ones.

I had hypothesized that the reason several previous studies had found no Allais paradox for small outcomes was that the high degree of risk-seeking for small outcomes made it impossible to express a change in risk preference using a choice technique. I did find that the Allais paradox was reduced in the small-outcome gambles when using single choice (but not choice titration), suggesting that the paradox may indeed be more difficult to detect using the choice technique. However, the Allais paradox was still significant for the small outcome gambles under single choice, which contradicts several previous studies that found no Allais paradox at all under these conditions. One possible explanation for this discrepancy is that in the present experiment, the value of highest outcome used in the single-choice questions was chosen specifically to increase the chances of finding the Allais paradox, based on the indifference points obtained during the choicetitration procedure. It is possible that individually tailoring the choices for each subject increased the percentage of subjects showing the Allais paradox beyond what was found in previous studies. It is also possible that making a somewhat lengthy series of previous choices during the matching section of the experiment also caused the subjects to be more prone to demonstrate the Allais paradox in single choice. Or perhaps it is merely a sign that a change in the Allais paradox for small outcomes is not a robust effect; Birnbaum (2004) also found the Allais paradox for gambles with comparatively small outcomes.

Regardless of these unanswered questions, the results of the present experiment call into question one important objection to the standard probability-weighting explanation of the Allais paradox. If the Allais paradox is caused by the $\pi$ function, it should occur for small outcomes as well as for large ones. Thus, past findings that the Allais paradox does not occur for small outcomes was not compatible with probability weighting. These results suggest that the Allais paradox still occurs for small outcomes, especially when more sensitive measures like choice titration are used. This result is compatible with probability-weighting theories.

\section{Probability Magnitude}

Choice titration. The mean indifference points for the gambles used in the probability manipulation are shown in Table 7. As for the outcome magnitude manipulation, higher indifference points for the CC-high gambles than for the CC-low gambles indicate the presence of the Allais paradox.

For each wave of testing, 203 subjects provided four indifference points, resulting in 812 total data points. In the first wave of the experiment, 56 data points (7\%) were missing due to failed check questions. These observations came from 39 subjects. In the second wave of the experiment, 59 data points $(7 \%)$ were missing due to failed check questions. These observations came from a total of 42 subjects. The missing data points were excluded from the analyses. Because there were no check questions for

Table 7

Probability Manipulation: Mean (With Standard Deviation) and Median (Med) Indifference Values for Highest Outcome, Before (Top) and After (Bottom) Responses at Floor and Ceiling Were Removed

\begin{tabular}{|c|c|c|c|c|c|c|c|}
\hline & \multirow{2}{*}{$\begin{array}{c}\text { Common } \\
\text { Consequence }\end{array}$} & \multicolumn{3}{|c|}{ High Probability } & \multicolumn{3}{|c|}{ Low Probability } \\
\hline & & $M$ & $S D$ & Med & $M$ & $S D$ & Med \\
\hline \multirow[t]{2}{*}{ Wave $1(1 / 2)$} & High & 51,558 & 29,988 & 30,859 & 40,362 & 23,829 & 26,172 \\
\hline & Low & 34,081 & 17,985 & 26,172 & 34,472 & 18,123 & 26,172 \\
\hline \multirow{3}{*}{ Wave $2(1 / 10)$} & High & 57,826 & 30,249 & 43,750 & 46,677 & 28,496 & 28,514 \\
\hline & Low & 34,554 & 16,934 & 27,344 & 34,339 & 18,221 & 26,172 \\
\hline & & \multicolumn{6}{|c|}{ No Floor/Ceiling } \\
\hline \multirow[t]{2}{*}{ Wave $1(1 / 2)$} & High & 54,524 & 25,661 & 44,921 & 49,825 & 22,980 & 40,234 \\
\hline & Low & 41,753 & 19,287 & 30,859 & 41,687 & 18,013 & 33,203 \\
\hline \multirow[t]{2}{*}{ Wave $2(1 / 10)$} & High & 60,778 & 25,024 & 56,640 & 57,922 & 25,594 & 54,296 \\
\hline & Low & 41,172 & 18,588 & 30,859 & 43,894 & 21,398 & 33,203 \\
\hline
\end{tabular}


the single choices, it was impossible for single-choice responses to produce missing values.

As shown in Table 7, the indifference points are higher for the CC-high gambles than for the CC-low gambles for both the large and small probabilities conditions. A 2 (common consequence present or absent) $\times 2$ (probability magnitude) ANOVA found that the main effect of common consequence was significant $[F(1,194)=49.20, p<$ .0001 , Wave $1 ; F(1,192)=103.48, p<.0001$, Wave 2], indicating an Allais paradox. Additionally, both the main effect of probability magnitude and the interaction between probability magnitude and common consequence were significant. ${ }^{3}$ [Probability magnitude: $F(1,196)=$ $10.52, p=.0014$, Wave $1 ; F(1,196)=10.54, p=.0014$, Wave 2. Interaction: $F(1,162)=12.10, p=.0006$, Wave 1 ; $F(1,160)=9.76, p=.0021$, Wave 2.] The main effect of probability magnitude indicates that subjects were more risk-seeking for small probabilities than they were for large ones. A reduction in risk aversion when probabilities are reduced by a common ratio is known as the common ratio effect and is a common finding in the risky-decision literature (e.g., Kahneman \& Tversky, 1979). The interaction indicates that the Allais paradox was smaller for small probabilities than it was for large ones, which is what we would expect from the probability-weighting explanation of the Allais paradox. However, although the Allais paradox was reduced in size when the probability was reduced, it was not eliminated: the simple main effect of common consequence was still significant for the low probability gambles $[F(1,178)=7.35, p=.0074$, Wave $1 ; F(1,179)=$ 25.54, $p<.0001$, Wave 2]. This is not predicted by probability-weighting theories. Fitting a multilevel model indicated that for Wave 1 (50\% probability), $11.2 \%$ of the explainable within-subjects variance was explained by the effects of common consequence and only $2.3 \%$ by the effects of probability magnitude. For Wave 2 (10\% probability), $20.4 \%$ of the explainable within-subjects variance was explained by the effects of common consequence and only $1.6 \%$ by the effects of probability magnitude. These results indicate that the presence or absence of the common consequence had a larger effect on indifference points than the probability magnitude.

Single choice. The percentage of subjects preferring the risky option for the single-choice questions is shown in Table 8. A 2 (common consequence present or absent) $\times 2$ (probability magnitude) logistic regression analysis found a significant Allais paradox $\left[\chi^{2}(1, N=203)=31.26, p<\right.$ .0001 , Wave $1 ; \chi^{2}(1, N=203)=22.39, p<.0001$, Wave 2] and also found that the Allais paradox was larger for large probabilities than for small ones $\left[\chi^{2}(1, N=203)=9.74\right.$, $p=.0018$, Wave $1 ; \chi^{2}(1, N=203)=21.61, p<.0001$, Wave 2]. In Wave 1 (50\% probability), the Allais paradox was still significant for the small probability gambles $\left[\chi^{2}(1, N=203)=8.00, p=.0047\right]$. In Wave $2(10 \%$ probability), it was not $\left[\chi^{2}(1, N=203)=0.71, p=.3394\right]$.

Probability magnitude summary. The standard demonstration of the Allais paradox has always used a certain amount as the safe CC-high gamble, and this certainty is an intrinsic part of the probability-weighting explanation of the paradox, which depends on the steepness at the ends of
Table 8

Probability Manipulation:

Percentage of Subjects Choosing the Risky Gamble

\begin{tabular}{|c|c|c|c|c|c|}
\hline & \multirow{2}{*}{$\begin{array}{c}\text { Common } \\
\text { Consequence }\end{array}$} & \multicolumn{2}{|c|}{$\begin{array}{c}\text { High } \\
\text { Probability }\end{array}$} & \multicolumn{2}{|c|}{$\begin{array}{c}\text { Low } \\
\text { Probability }\end{array}$} \\
\hline & & $\%$ & $n$ & $\%$ & $n$ \\
\hline \multirow[t]{2}{*}{ Wave $1(1 / 2)$} & High & 62 & 126 & 71 & 145 \\
\hline & Low & 87 & 177 & 81 & 165 \\
\hline \multirow[t]{2}{*}{ Wave $2(1 / 10)$} & High & 57 & 115 & 70 & 142 \\
\hline & Low & 84 & 171 & 73 & 149 \\
\hline
\end{tabular}

Table 9

Probability Manipulation:

Percentage of Responses at Floor/Ceiling

\begin{tabular}{|c|c|c|c|c|c|c|c|c|c|}
\hline & \multirow{3}{*}{$\begin{array}{c}\text { Common } \\
\text { Consequence }\end{array}$} & \multicolumn{4}{|c|}{ High Probability } & \multicolumn{4}{|c|}{ Low Probability } \\
\hline & & \multicolumn{2}{|c|}{ Floor } & \multicolumn{2}{|c|}{ Ceiling } & \multicolumn{2}{|c|}{ Floor } & \multicolumn{2}{|c|}{ Ceiling } \\
\hline & & $\%$ & $n$ & $\%$ & $\bar{n}$ & $\%$ & $n$ & $\%$ & $n$ \\
\hline \multirow[t]{2}{*}{ Wave $1(1 / 2)$} & High & 34 & 63 & 15 & 28 & 52 & 96 & 6 & 11 \\
\hline & Low & 61 & 118 & 3 & 6 & 60 & 116 & 4 & 7 \\
\hline \multirow[t]{2}{*}{ Wave $2(1 / 10)$} & High & 27 & 48 & 17 & 30 & 49 & 92 & 11 & 20 \\
\hline & Low & 50 & 98 & 2 & 3 & 60 & 117 & 2 & 4 \\
\hline
\end{tabular}

the $\pi$ function. When the gambles are shifted far from the steep endpoints of the $\pi$ function and into the flat middle regions, the Allais paradox should vanish. This is not what was found in the present experiment. To be consistent with the present results, the $\pi$ function would have to be convex everywhere (see Figure 2), rather than in the commonly proposed inverse-S shape. A convex $\pi$ function is consistent with the requirements imposed on the shape of the $\pi$ function by original PT (Kahneman \& Tversky, 1979) but contradicts more recent experimental results (e.g., Camerer \& Ho, 1994; Gonzalez \& Wu, 1999; Tversky \& Kahneman, 1992; Wu \& Gonzalez, 1996, 1998), which support the inverse-S shaped $\pi$ function.

The distribution of responses at floor and ceiling was particularly interesting (see Table 9). For the high-probability gambles, where the safe CC-high gamble was certain, far more responses showed a ceiling effect for the CC-high gambles than for the $\mathrm{CC}$-low gambles. This indicates a high

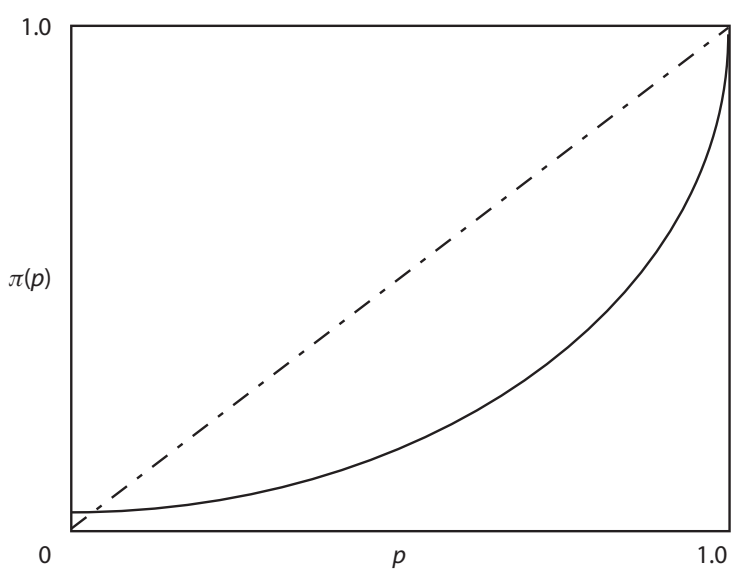

Figure 2. A $\pi$ function consistent with an Allais paradox for small probabilities. 
level of risk aversion, suggesting that the subject preferred the safe gamble no matter how high the highest outcome became. For the low-probability gambles, this tendency was less pronounced. The number of floor responses (indicating extreme risk-seeking) was greater for the lowprobability gambles than for the high-probability ones, especially for the CC-high gambles. This suggests that the Allais paradox may not be fully accounted for by a special appeal to certainty, but that the presence of certainty may inhibit extreme risk-seeking and encourage extreme risk aversion. The interaction between probability magnitude and common consequence was no longer significant after all responses showing extreme risk-seeking or risk aversion were removed. This may indicate that the reduction of the Allais paradox for reduced probabilities was driven entirely by the decrease in the tendency to show extreme risk preferences. However, it is also possible that the failure to find a significant interaction was merely due to reduced power because of the decrease in the number of data points after floor and ceiling responses were removed.

\section{DISCUSSION}

The outcome of the present experiment suggests that the effects of payout and probability magnitude on the Allais paradox are the opposite of what they have generally been assumed to be. Although some past experiments had found that the Allais paradox did not occur for gambles with small outcomes, the present experiment did not find this to be the case; the paradox occurred even for gambles with quite small outcomes. Although past theories have always assumed that drastically reducing the nonzero outcomes of Allais paradox gambles would eliminate the paradox, the present experiment found that not to be the case, either; the Allais paradox was still present, although reduced in size, even when the probabilities were quite drastically reduced.

\section{The Allais Paradox and Probability Weighting}

There are many theories of risky choice that posit a probability-weighting function. Two of these theories, original PT and cumulative PT, have been the leading descriptive theories of risky choice for many years.

The present experiment provides some support for these theories. As mentioned above, past findings that the Allais paradox does not occur for small outcomes are not consistent with the standard $\pi$ function explanation of the paradox. Using a more sensitive technique than previous experiments did, the present experiment found the Allais paradox for both large and small outcomes. There is some suggestion that the size of the Allais paradox was slightly reduced when outcomes were decreased by a factor of 5,000 . However, a small decrease in the size of the Allais paradox when outcome magnitudes are greatly reduced can be explained by the shape of the utility function, and thus is not problematic for probability-weighting theories. Therefore, the present findings for the effects of outcome magnitude present no difficulty for the leading explanations of the Allais paradox.

However, the findings concerning probability magnitude are more problematic for probability-weighting theo- ries. The assumption of all probability-weighting theories is that the Allais paradox is essentially a certainty effect, depending on a very steep shape of the $\pi$ function near the endpoints. Under this account, the paradox can occur in the absence of certainty only if the probabilities used capitalize on the nonlinearity of the $\pi$ function. As discussed above, the standard Allais paradox is easily explained by the inverse-S shaped $\pi$ function supported by the literature (e.g., Camerer \& Ho, 1994; Gonzalez \& Wu, 1999; Tversky \& Kahneman, 1992; Wu \& Gonzalez, 1996, 1998). However, a $\pi$ function that explains the present results would have to be convex everywhere, with an accelerating slope, as shown in Figure 2. Assuming we are unwilling to accept a $\pi$ function consistent with the present results but inconsistent with the wider literature, probability-weighting theories are not consistent with the finding that the Allais paradox occurred for the small-probability outcomes.

\section{Other Theories of the Allais Paradox}

Although probability-weighting explanations are the most common explanations of the Allais paradox, other explanations exist, of which I will discuss two: configuralweight theories and decision-affect theories.

In configural-weight theories, the weight an outcome receives depends on how good the outcome is relative to the other possible outcomes in the gamble. One example of a configural weight model is Birnbaum's transfer of attentional exchange (TAX) model (Birnbaum, 1997, 1999). In this model, probability weight is transferred from better outcomes to worse outcomes as more outcomes are added. Essentially, each outcome "taxes" the probability of every better outcome, increasing its own weight at the expense of the better outcomes. This captures the intuition that decision makers often focus disproportionately on the least favorable outcome of a gamble.

The TAX model explains the Allais paradox via the transfer of probability weight from the highest outcome to the lowest outcome in the risky CC-high gamble. The $\$ 0$ outcome in this gamble taxes weight from both the middle and highest outcome, and thus receives more weight than the probability alone would warrant, whereas the highest outcome loses probability weight. Thus, decision makers prefer the safe gamble when choosing between the $\mathrm{CC}$-high gambles. However, in the CC-low gambles, both gambles have two outcomes. Thus, the probability weights undergo similar changes in both gambles and decisionmakers simply choose the higher paying gamble, which is the risky gamble.

Configural-weight models like the TAX model are consistent with the outcome magnitude results of the present experiment. However, under parameters fitting previous data in the literature (e.g., Birnbaum, 1997, 1999), the TAX model does not predict the probability weighting results. It is true that even when the probabilities have been reduced the risky CC-high gamble has more outcomes than the safe CC-high gamble, and this may be related to the continued presence of the Allais paradox for the reducedprobability gambles. However, according to a TAX model using parameters from previous experiments (Birnbaum \& Bailey, 1998), subjects should not have exhibited the 
Allais paradox for the small-probability gambles; rather, they should have chosen the risky gamble in both the CChigh and CC-low gamble pairs.

Decision-affect theories propose that some decisions are best explained by the emotions associated with the decision. Perhaps the most intuitively compelling explanation of the Allais paradox involves the emotions associated with losing the risky CC-high gamble. Suppose you have the chance to play the standard form of the Allais paradox ( $10 \%$ chance of $\$ 5$ million; $89 \%$ of $\$ 1$ million; $1 \%$ of $\$ 0$ vs. $100 \%$ of $\$ 1$ million) and choose to take the risky gamble. You reach in to draw a ball out of the urn and draw the one ball that results in a $\$ 0$ outcome. Although you are actually no worse off than you were before, you would probably be disappointed. After all, if you had only chosen the safe option instead of the risky one, or received any other ball but that one, you would now be rich!

Intuitively, such a situation seems to play a large part in a decision maker's preference for the safe CC-high gamble over the risky CC-high gamble. These intuitions are captured by decision-affect theories, a group of theories that have not been well studied in relation to the Allais paradox. These theories propose that people's decision-making behavior is driven by the emotions they feel (or anticipate feeling) in connection with the consequences of the decision, usually either disappointment or regret. Disappointment refers to the emotion experienced when a different state of the world would have produced a better outcome: "If only it had been a different ball!" Regret is the emotion experienced when a different choice on your part would have produced a better outcome: "If only I had picked the safer gamble!" Examples of such theories include disappointment theory (Bell, 1985; Loomes \& Sugden, 1986), regret theory (Bell, 1982; Loomes \& Sugden, 1982), and decision-affect theory (Mellers, Schwartz, Ho, \& Ritov, 1997).

The decision-affect explanation of the Allais paradox depends on the possibility of experiencing negative emotions related to the CC-high gamble. In the CC-high gamble, the disappointment and/or regret that decision makers would experience after receiving the $\$ 0$ outcome is so large that they prefer to eliminate this possibility altogether by choosing the safe CC-high gamble. In the CC-low gambles, the probability of losing is high for both the safe and risky gambles. Decision-affect theories propose that disappointment is reduced when the probability of winning the chosen gamble is low, and that regret is reduced when the probability of winning the gamble not chosen is low. Thus, for the CC-low gambles, the potential for negative affect is reduced and is roughly the same for both gambles. Freed from emotional considerations, decision makers choose the CC-low gamble with the higher outcome.

Although these theories are intuitively compelling, they are not consistent with the results of the present experiment. Both theoretically and empirically, disappointment and regret are smaller when outcomes are smaller-no one is particularly upset to lose a gamble for a $\$ 5$ prize (e.g., Mellers et al., 1997; Mellers, Schwartz, \& Ritov, 1999; van Dijk \& van der Pligt, 1997). If disappointment or regret were responsible for the Allais paradox, we would expect the paradox to be smaller for small out- comes, and probably disappear altogether for very small outcomes. This is consistent with some of the previous findings in the literature, but not with the present results, which found a smaller Allais paradox for small outcomes only for single choice, not with the more sensitive matching technique. Decision-affect theories also do not address the results of the probability-magnitude manipulation. Although decision-affect theories are consistent with the reduction of the Allais paradox for small probabilities, they do not explain why the paradox is not eliminated even when losing is very likely for both gambles, and thus disappointment and regret are not considerations in choosing between them.

After conducting experimental tests of multiple theories of decision making, Camerer (1989) observed that no single theory can explain all the decision biases and inconstancies shown by decision makers. This could equally well be applied to the results of the present experiment: I am aware of no theory that can explain both the probability-magnitude and outcome-magnitude data while remaining consistent with the wider literature. The continued presence of the Allais paradox when the probability of the nonzero outcomes was reduced is very surprising. Though it is not yet clear how robust the phenomenon is, it has now been shown in three groups of subjects (the pilot study as well as in both groups in the present experiment), with both choice and matching responses, and with both pencil-and-paper and Web-based responses. This suggests that the effects of probability on the Allais paradox merit further investigation, to determine the boundaries within which reduced probability common-consequence effects can occur. Additional research, perhaps utilizing exit interviews or process tracing techniques, may also yield more insight into the decision processes that result in the reduced-probability Allais paradox, and whether they are the same processes involved in the standard full-probability Allais paradox.

Besides pointing to the need for further research, the results of the present experiment have implications for the field of decision research. The Allais paradox has long been assumed to be a form of the certainty effect, and has been used as an example of the certainty effect experimentally (e.g., Keren \& Roelofsma, 1995). The present results suggest that the Allais paradox is not strictly speaking a certainty effect, and may produce different experimental results than do other biases thought to be forms of the certainty effect (e.g., Weber \& Chapman, 2005a). Using the Allais paradox as a certainty effect in experiments may thus generate misleading results concerning the nature of certainty.

If the Allais paradox is not simply a form of the certainty effect, what does cause the paradox to occur? Answering that question will require additional research into the nature and limitations of the reduced-probability Allais paradox. It seems that after 60 years of research, the Allais paradox may yet remain as it was when first proposed: a challenge to leading theories of decisionmaking.

\section{AUTHOR NOTE}

This article is based on B.J.W.'s doctoral dissertation and was supported by an NSF graduate fellowship to the author. B.J.W. thanks her dissertation advisor, Dr. Gretchen Chapman, for her comments on the 
manuscript, experimental design, and analysis, and Dr. Scott Huettel for his comments on the manuscript. Correspondence concerning this article should be addressed to B. J. Weber, Brain Imaging and Analysis Center, Duke University Medical Center, P.O. Box 3918, Durham, NC 27710 (e-mail: weber@biac.duke.edu).

\section{REFERENCES}

Allais, M. (1953). Le comportement de l'homme rationnel devant le risque: Critique des postulats et axiomes de l'école américaine. Econometrica, 21, 503-546.

BELL, D. E. (1982). Regret in decision making under uncertainty. Operations Research, 30, 961-981.

BeLL, D. E. (1985). Disappointment in decision making under uncertainty. Operations Research, 33, 1-27.

Birnbaum, M. H. (1997). Violations of monotonicity in judgment and decision making. In A. A. J. Marley (Ed.), Choice, decision, and measurement: Essays in honor of R. Duncan Luce (pp. 73-100). Mahwah, NJ: Erlbaum.

BIRNBAUM, M. H. (1999). The paradoxes of Allais, stochastic dominance, and decision weights. In J. Shanteau, B. A. Mellers, \& D. A. Schum (Eds.), Decision science and technology: Reflections on the contributions of Ward Edwards (pp. 27-52). Norwell, MA: Kluwer.

Birnbaum, M. H. (2004). Causes of Allais common consequence paradoxes: An experimental dissection. Journal of Mathematical Psychology, 48, 87-106.

BirNBAUM, M. H. (2007). Tests of branch splitting and branch-splitting independence in Allais paradoxes with positive and mixed consequences. Organizational Behavior \& Human Decision Processes, 102, 154-173.

Birnbaum, M. H., \& Bailey, R. (1998). Taxcalculator: Configural weight, TAX model and cumulative prospect model [Computer software]. Retrieved from http://psych.fullerton.edu/mbirnbaum/calculators/TAXcalculator.htm.

Camerer, C. F. (1989). An experimental test of several generalized utility theories. Journal of Risk \& Uncertainty, 2, 61-104.

Camerer, C. F. (1992). Recent tests of generalizations of expected utility theory. In W. Edwards (Ed.), Utility theories: Measurements and applications (pp. 207-252). Boston: Kluwer.

CAMERer, C. F., \& Ho, T. H. (1994). Violations of the betweenness axiom and nonlinearity in probability. Journal of Risk \& Uncertainty, 8, 167-196.

Chapman, G. B., \& Weber, B. J. (2006). Decision biases in intertemporal choice and choice under uncertainty: Testing a common account. Memory \& Cognition, 34, 589-602.

Chew, S. H., \& WALLER, W. S. (1986). Empirical tests of weighted utility theory. Journal of Mathematical Psychology, 30, 55-72.

Conlisk, J. (1989). Three variants on the Allais example. American Economic Review, 79, 392-407.

Du, W., Green, L, \& Myerson, J. (2002). Cross-cultural comparisons of discounting delayed and probabilistic rewards. Psychological Record, 52, 479-492.

FAN, C.-P. (2002). Allais paradox in the small. Journal of Economic Behavior \& Organization, 49, 411-421.

Green, L., Myerson, J., \& Ostaszewski, P. (1999). Amount of reward has opposite effects on the discounting of delayed and probabilistic outcomes. Journal of Experimental Psychology: Learning, Memory, \& Cognition, 25, 418-427.

Gonzalez, R., \& Wu, G. (1999). On the shape of the probability weighting function. Cognitive Psychology, 38, 129-166.

HarLess, D. (1992). Predictions about indifference curves inside the unit triangle: A test of variants of expected utility theory. Journal of Economic Behavior \& Organization, 18, 391-414.

Kahneman, D., \& TVERSKY, A. (1979). Prospect theory: An analysis of decision under risk. Econometrica, 47, 263-291.

Keren, G., \& Roelofsma, P. (1995). Immediacy and certainty in intertemporal choice. Organizational Behavior \& Human Decision Processes, 63, 287-297.

LichtensteIN, S., \& SLOvic, P. (1971). Reversals of preference between bids and choices in gambling decisions. Journal of Experimental Psychology, 89, 46-55.
Loomes, G., \& SUgden, R. (1982). Regret theory: An alternative theory of rational choice under uncertainty. Economic Journal, 92, 805-824.

Loomes, G., \& Sugden, R. (1986). Disappointment and dynamic consistency in choice under uncertainty. Review of Economic Studies, 53, 271-282.

Machina, M. (1982). "Expected utility" analysis without the independence axiom. Econometrica, 50, 277-323.

MARSCHAK, J. (1950). Rational behavior, uncertain prospects and measurable utility. Econometrica, 18, 111-141.

Mellers, B., Schwartz, A., Ho, K., \& Ritov, I. (1997). Decision affect theory: Emotional responses to the outcomes of risk gambles. Psychological Science, 8, 423-428.

Mellers, B., Schwartz, A., \& Ritov, I. (1999). Emotion-based choice. Journal of Experimental Psychology: General, 128, 332-345.

Myerson, J., Green, L., Hanson, J. S., Holt, D. D., \& Estle, J. S. (2003). Discounting delayed and probabilistic rewards: Processes and traits. Journal of Economic Psychology, 24, 619-635.

QuigGin, J. (1982). A theory of anticipated utility. Journal of Economic Behavior \& Organization, 3, 323-343.

Rachlin, H., Brown, J., \& Cross, J. (2000). Discounting in judgments of delay and probability. Journal of Behavioral Decision Making, 13, 145-159.

SAVAGE, L. J. (1954). The foundations of statistics. New York: Wiley,

SLovic, P., \& Lichtenstein, S. (1983). Preference reversals: A broader perspective. American Economic Review, 73, 596-605.

SLovic, P., \& TVERSKY, A. (1974). Who accepts Savage's axiom? Behavioral Science, 19, 368-373.

Sopher, B., \& Gigliotti, G. (1993). A test of generalized expected utility theory. Theory \& Decision, 35, 75-106.

TVersky, A., \& Kahneman, D. (1992). Advances in prospect theory: Cumulative representation of uncertainty. Journal of Risk \& Uncertainty, 5, 297-323.

Tversky, A., Slovic, P., \& Kahneman, D. (1990). The causes of preference reversal. American Economic Review, 80, 204-217.

VAN DIJK, W., \& VAN DER PLIGT, J. (1997). The impact of probability and magnitude of outcome on disappointment and elation. Organizational Behavior \& Human Decision Processes, 69, 277-284.

Weber, B., \& Chapman, G. (2005a). The combined effect of risk and time on choice: Does uncertainty eliminate the immediacy effect? Does delay eliminate the certainty effect? Organizational Behavior \& Human Decision Processes, 96, 104-118.

Weber, B., \& Chapman, G. (2005b). Playing for peanuts: Why is risk seeking more common for low-stakes gambles? Organizational Behavior \& Human Decision Processes, 97, 31-46.

Weber, B., Marks, M., \& Chapman, G. (2003, November). Variants on the Allais paradox: Testing the certainty effect account. Poster session presented at the annual meeting of the Society for Judgment and Decision Making, Vancouver, BC.

Wu, G., \& GonZALEZ, R. (1996). Curvature of the probability weighting function. Management Science, 42, 1676-1690.

Wu, G., \& Gonzalez, R. (1998). Common consequence conditions in decision making under risk. Journal of Risk \& Uncertainty, 16, 115-139.

\section{NOTES}

1. The Friedman test indicated that the effect of outcome magnitude on Allais paradox size was significant in Wave $2\left[\chi^{2}(1, N=357)=\right.$ $7.57, p=.0059]$.

2. The multilevel analysis indicated that the effect of outcome magnitude on Allais paradox size was significant in Wave $2[F(1,549)=$ $4.47, p=.03]$.

3. When all responses that were at either floor or ceiling were removed, neither the main effect of probability magnitude nor the interaction between probability magnitude and common consequence was significant in either wave of the experiment. [Probability magnitude: $F(1,78)=$ $0.90, p=.35$, Wave $1 ; F(1,88)=0.00, p=.98$, Wave 2. Interaction: $F(1,31)=0.85, p=.36$, Wave $1 ; F(1,27)=1.26, p=.27$, Wave 2.]

(Manuscript received October 10, 2006; revision accepted for publication February 4, 2008.) 